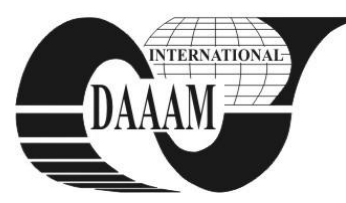

Annals of DAAAM for 2011 \& Proceedings of the 22nd International DAAAM Symposium, Volume 22, No. 1, ISSN 1726-9679 ISBN 978-3-901509-83-4, Editor B. Katalinic, Published by DAAAM International, Vienna, Austria, EU, 2011 Make Harmony between Technology and Nature, and Your Mind will Fly Free as a Bird Annals \& Proceedings of DAAAM International 2011

\title{
MULTIAGENT ROBOTIC COLLABORATIVE FRAMEWORK
}

\author{
SVACO, M[arko]; SEKORANJA, B[ojan] \& JERBIC, B[ojan]
}

\begin{abstract}
A cooperative multiagent robotic framework for industrial assembly applications is presented. Absolute positioning in robotics is a very demanding topic. A method has been developed for spatial calibration of multiple robots. A multiagent system architecture has been developed where robots provide and request particular services from other participants of the multiagent system. Those services include manipulation, pick, place, transport and etc. Visual feedback is used as the main tool for providing highly accurate relative positioning between multiple robots in the environment. The framework is implemented on an actual multi-robot setup.
\end{abstract}

Key words: multiagent robotic cooperation, spatial calibration

\section{INTRODUCTION}

In the dynamic global economy aspects of production and assembly technologies are rapidly changing. High batch production is being replaced with numerous variants of products tailored for specific customer demands. Mass customization (Hales, 1992) has replaced mass production. Highly responsive (Seilonen, 2009) and flexible systems need to replace single-purpose machines and production lines that address only specific products. The system hierarchy and control methods need to adapt to these requirements. Multiagent systems (Wooldridge, 2002) exhibit characteristics that are beneficial and highly applicable for such applications. Inherently distributed, with the property of operating without need for central control; and self-organization are main attributes that can be utilized for control of flexible production and assembly systems.

Today, multiagent industrial applications range from resource scheduling (Pirttioja, 2005) to applications of selforganizing assembly systems (Frei et al., 2008). It has been proven that the multiagent control concept and its' architecture can provide a foundation for flexible, adaptive and semiautonomous industrial systems.

The presented work particularly addresses issues involving multiagent robotic assembly systems (Svaco et al., 2010). System components are treated as autonomous entities with defined knowledge and cognition of other agents and their environment. Every agent can provide and request specific services, e.g. manipulation, pick and place operations, transportation and etc. Through these abstract concepts all components in the system communicate, interact and organize toward accomplishing a common global goal.

The initial problem in application of service oriented architecture is the spatial calibration of multiple robots in their work environment. In this paper a novel method for spatial calibration and relative positioning of multiple robots is presented along with the fundamental principles of multiagent services.

The proposed solution utilizes a visual method for relative positioning. The calibration method and multiagent services are detailed in the following sections.

\section{MULTI ROBOT CALIBRATION MEHOD}

Price of industrial robots compared to their ability of performing more complex tasks with even higher precision is decreasing. Cumulative absolute accuracy of multiple robotic units is a complex issue and is difficult to attain at a desirable level for precise positioning and delicate assembly operations. Through various experiments and available multiple robot calibration techniques and methods desirable accuracy wasn't obtained.

The proposed calibration method utilizes a two step approach. First part comprises a coarse spatial calibration of multiple robots $R_{n}(n=1 \ldots a)$. Robot tool centre points (TCP) are guided to a desired position in the shared workspace. A set of global Cartesian coordinate systems $C_{m}(m=1 \ldots b)$ are acquired with the following parameters: $C_{m}=\left\{R_{l}, R_{2} \ldots R_{n}\right\} . R_{n}$ depicts a set of local coordinates accessible in $C_{m}$ to the current robot. Obtained absolute positioning error is one order higher compared to a single robotic agent and cannot provide desired precision. Calibration information is written as global knowledge for each robot and is used in the visual calibration step. A schematic view of the visual method for error correction is depicted in Fig. 1. Shared robot workspaces are divided in spatial quadrants with inherent errors provided by the initial calibration. Each quadrant is a rectangular cube with a side length of $100 \mathrm{~mm}$. In these quadrants coarse positioning of robot TCP's is achieved. Points P1 and P1' reached by Robot 1 and Robot 2 prior to the visual calibration are depicted in Fig. 1 a). Accurate positioning is established using visual feedback by acquiring relative positions of robot TCP's. Visual markers are used for this procedure. Robot 2 visually identifies the relative position of Robotl and acquires that information. A new coordinate system is calculated with an offset about the initial system. Robot 2 corrects its position as shown in Fig. 1 b). The spatial calibration of two robots for the current quadrant is subsequently written in a 3D matrix as a correction index for the current quadrant. All further tasks in calibrated quadrants utilize the correction index.

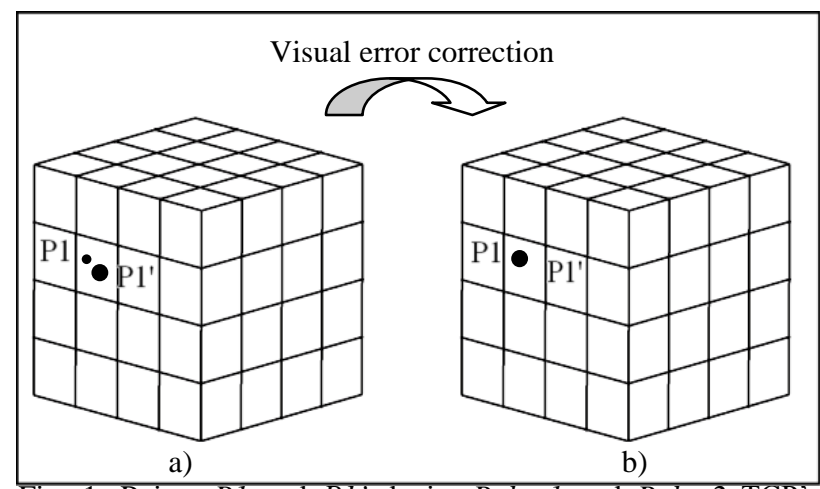

Fig. 1. Points $P I$ and $P I$ ' depict Robotl and Robot2 TCP's respectably a) Positioning error prior to visual calibration b) Corrected positioning error after visual calibration 


\section{SERIVCE ORIENTED ARCHITECTURE}

For explicit multiagent robot programming a service oriented multiagent architecture was developed. System components i.e. agents are self-aware entities capable of decision making and negotiating with other agents. For complex and adaptive assembly systems this methodology opens new possibilities and boosts productivity in unpredictable production conditions. Fig. 2 depicts a schematic representation of the service oriented multiagent robotic architecture. Agents in the system are collaborative entities and have a common global goal (product assembly). By following the general assembly plan (GAP) agents are familiarized with all relevant assembly information. The GAP comprises part, product and assembly process information. The assembly sequence is written as a set of abstract steps that can be translated into specific tasks within a single agent's local plan. The GAP is a notation of the assembly sequence and does not take into consideration a particular agent for providing a service or accomplishing a task. Therefore the GAP is coded in a comprehensible way and can be interpreted for any given agent in any given initial state. By inspecting their actual status, the state of the environment and current process stage an agent reasons about further actions. Tasks can be performed either individually of by requesting additional agents for support in form of multiagent services (Fig. 2). Agents initially request the nearest idle neighbor for a specific service; if this is not plausible a request for a service is appended on the multiagent virtual blackboard (MVB) and the best bid is accepted.

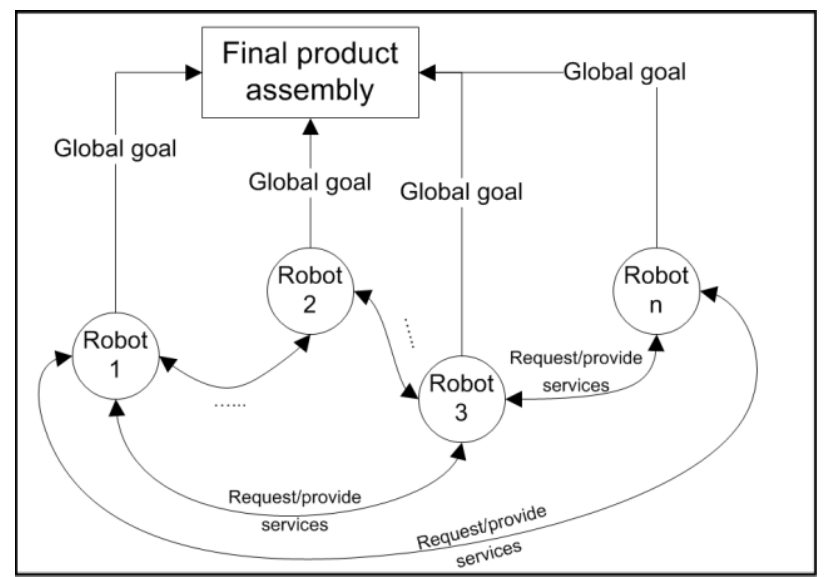

Fig. 2. Service oriented architecture

The set of multiagent services include:

- $\quad$ Pick (pick_position, $C_{m}$ )

- $\quad H o l d$ (hold_position, $C_{m}$ )

- Transport (initial_postion, final_position, $C_{m}$ )

- Reorient (initial_orientation,final_orientation, $C_{m}$ )

- Assemble (assembly_position, assembly_operation, assembly_parameters, $C_{m}$ )

Through these services new actions emerge and tasks with higher complexity can be achieved. If an agent needs a specific part defined in the GAP and that part is not currently in its' workspace it requests this part using the Transport service. An agent that can transport the part accepts the service call. If necessary visual calibration is performed and the service is adequately accomplished.

\section{RESULTS}

The framework has been tested on an actual system (Fig. 3.) consisting of 4 industrial robots. The calibration steps presented in section 2 were implemented. Initial tests provided desired accuracy where the order of reproduced error in multi- robot handling tasks and interaction was approximate to the accuracy of a single robot. The main limitation of the developed approach is extended process cycle time. This is influenced by the calibration procedure as every non calibrated quadrant requires visual calibration. High level of reactive and flexible control affects overall system efficiency.

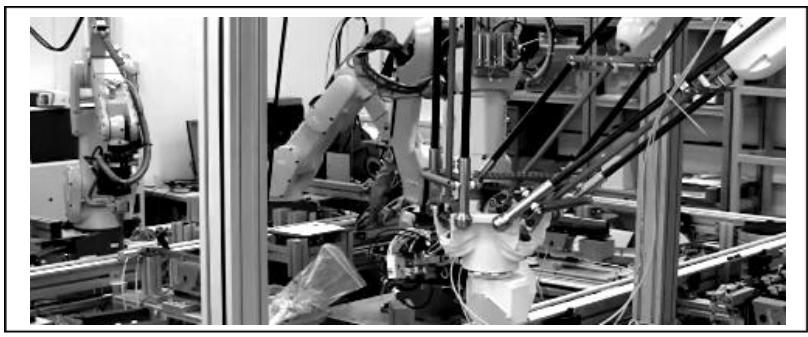

Fig. 3. Actual robotic setup

\section{CONCLUSION AND FURTHER WORK}

A brief insight into the developed methodology of service oriented architecture and the method of spatial visual calibration has been presented. The service oriented architectures is a functional addition of previous work (Svaco et al., 2011) and a step toward practical autonomous and intelligent assembly robotic systems. Conventional assembly system organization is oriented toward one product or a small number of variants. For a system to be highly responsive toward market demands various mechanisms of reactive and adaptive behavior need to be embedded in the design stage. In such complex environments all actions and their respective responses cannot be predefined. System components (agents) need to find solutions in a collaborative fashion. The developed system can solve problems which emerge from the presence of deterministic chaos, unpredictable assembly conditions and dynamic changes in market demands.

\section{REFERENCES}

Frei, R.; Di Marzo Serugendo, G. \& Barata, J. (2008). Designing self-organization for evolvable assembly systems. Proceedings of Int. Conf. on Self-Adaptive and Self-Organizing Syst. (SASO), Brueckner S., Robertson P. \& Bellur U. (Ed.),Venice, pp. 97-106, IEEE

Hales H. L. (1992). Automating and Integrating the Sales Function: How to Profit From Complexity and Customization. Enterprise Integration Strategies, Vol. 9, No. 11, pp. 1-9

Pirttioja, T.; Pakonen, A.; Seilonen, I.; Halme, A. \& Koskinen, K. (2005). Multi-agent based information access services for condition monitoring in process automation. Proc. of The 3rd International IEEE Conference on Industrial Informatics (INDIN 2005). Perth, Australia, IEEE

Seilonen, I.; Pirttioja, T. \& Koskinen, K. (2009). Extending process automation systems with multiagent techniques. Engineering Applications of Artificial Intelligence, Vol. 22, Issue 7, (October, 2009), pp. 1056-1067, ISSN: 0952-1976

Svaco M.; Sekoranja B. \& Jerbic B. (2010).A Multiagent approach for development of a flexible and adaptive robotic assembly work cell, Proc. of The 3rd Int. Conf. on Computational Intelligence and Industrial Application (PACIIA 2010), Zhang, Y. ; Tan, H. (Ed.), pp. 64-67, IEEE

Svaco M.; Sekoranja B. \& Jerbic B. (2011). Autonomous Planning Framework for Distributed Multiagent Robotic Systems, Proc. of The 2nd Doctoral Conf. on Computing, Electrical and Industrial Syst. DoCEIS 2011, Costa de Caparica, Portugal, Luis M. Camarinha-Matos (Ed.), pp. 147-154, Springer, Heidelberg

Wooldridge, M. (2002). An Introduction to Multiagent Systems, John Wiley \& Sons, 047149691X, Chichester, England 